\title{
Gene expression profile after cardiopulmonary bypass and cardioplegic arrest
}

\author{
Marc Ruel, MD, MPH \\ Cesario Bianchi, MD, PhD \\ Tanveer A. Khan, $\mathrm{MD}^{\mathrm{a}}$ \\ Shu Xu, PhD \\ John R. Liddicoat, $M D^{\mathrm{a}}$ \\ Pierre Voisine, $M D^{\mathrm{a}}$ \\ Eugenio Araujo, $\mathrm{PhD}^{\mathrm{a}}$ \\ Helen Lyon, MD ${ }^{\mathrm{b}}$ \\ Isaac S. Kohane, $\mathrm{MD}, \mathrm{PhD}^{\mathrm{c}}$ \\ Towia A. Libermann, $\mathrm{PhD}^{\mathrm{d}}$ \\ Frank W. Sellke, MDa
}

Objective: This study examines the cardiac and peripheral gene expression responses to cardiopulmonary bypass and cardioplegic arrest.

Methods: Atrial myocardium and skeletal muscle were harvested from 16 patients who underwent coronary artery bypass grafting before and after cardiopulmonary bypass and cardioplegic arrest. Ten sample pairs were selected for patient similarity, and oligonucleotide microarray analyses of 12,625 genes were performed using matched precardiopulmonary bypass tissues as controls. Array results were validated with Northern blotting, real-time polymerase chain reaction, in situ hybridization, and immunoblotting. Statistical analyses were nonparametric.

From the Division of Cardiothoracic Surgery, Beth Israel Deaconess Medical Center $^{\mathrm{a}}$ and Harvard Medical School, and Divisions of Medical Genetics ${ }^{\mathrm{b}}$ and Informatics Program, ${ }^{\mathrm{c}}$ Children's Hospital and Harvard Medical School, and the Genomics Center, ${ }^{\mathrm{d}}$ Beth Israel Deaconess Medical Center and Harvard Medical School, Boston, Mass.

This work was funded by grant HL-46716 (Dr Sellke) and R24 DK58739 (Drs Libermann and Kohane) from the National Institutes of Health. Dr Ruel is supported by postdoctoral research awards from the Heart and Stroke Foundation of Canada and the Canadian Institutes of Health Research.

Received for publication Dec 5, 2002; revisions requested Jan 21, 2003; revisions received Feb 5, 2003; accepted for publication March 11, 2003.

Address for reprints: Frank W. Sellke, MD, Division of Cardiothoracic Surgery, Beth Israel Deaconess Medical Center, LMOB

2A, 110 Francis St, Boston, MA 02215 (E-mail: fsellke@bidmc.harvard.edu).

J Thorac Cardiovasc Surg 2003;126: $1521-30$

Copyright $(92003$ by The American Association for Thoracic Surgery

$0022-5223 / 2003 \$ 30.00+0$

doi:10.1016/S0022-5223(03)00969-3
Results: Median durations of cardiopulmonary bypass and cardioplegic arrest were 74 and 60 minutes, respectively. Compared with precardiopulmonary bypass, postcardiopulmonary bypass myocardial tissues revealed 480 up-regulated and 626 down-regulated genes with a threshold $P$ value of .025 or less (signal-to-noise ratio: 3.46); skeletal muscle tissues showed 560 and 348 such genes, respectively (signalto-noise ratio: 3.04$)$. Up-regulated genes in cardiac tissues included inflammatory and transcription activators FOS; jun B proto-oncogene; nuclear receptor subfamily 4, group A, member 3; MYC; transcription factor-8; endothelial leukocyte adhesion molecule-1; and cysteine-rich 61; apoptotic genes nuclear receptor subfamily 4, group $\mathrm{A}$, member 1 and cyclin-dependent kinase inhibitor $1 \mathrm{~A}$; and stress genes dual-specificity phosphatase-1, dual-specificity phosphatase-5, and B-cell translocation gene 2. Up-regulated skeletal muscle genes included interleukin 6; interleukin 8; tumor necrosis factor receptor superfamily, member $11 \mathrm{~B}$; nuclear receptor subfamily 4 , group A, member 3 ; transcription factor-8; interleukin 13 ; jun B proto-oncogene; interleukin $1 \mathrm{~B}$; glycoprotein $\mathrm{Ib}$, platelet, alpha polypeptide; and Ras-associated protein RAB27A. Down-regulated genes included haptoglobin and numerous immunoglobulins in the heart, and factor H-related gene 2, protein phosphatase 1 , regulatory subunit $3 \mathrm{~A}$, and growth differentiation factor- 8 in skeletal muscle.

Conclusions: By establishing a profile of the gene-expression responses to cardiopulmonary bypass and cardioplegia, this study allows a better understanding of their effects and provides a framework for the evaluation of new cardiac surgical modalities directly at the genome level. 


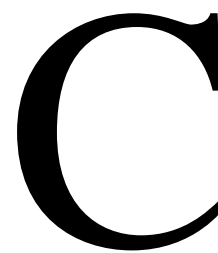

ardiopulmonary bypass (CPB) and cardioplegic arrest are used in more than 300,000 patients who undergo cardiac surgery in the United States every year. Although off-pump coronary surgery has recently gained in popularity, CPB and cardioplegic arrest are still employed in more than $75 \%$ of coronary artery bypass grafting $(\mathrm{CABG})$ procedures and for virtually every other type of adult cardiac operation. Although these modalities are indispensable adjuncts, they are also paradoxically responsible for a large part of the morbidity associated with cardiac surgery. CPB is associated with disturbances of virtually every organ system, mediated by its elicitation of a whole-body inflammatory response. ${ }^{1-4}$ Cardioplegic arrest may result in coronary vasomotor dysfunction, ischemia-reperfusion injury, myocardial edema, perioperative myocardial infarction, and low cardiac output syndrome. ${ }^{5-7}$

Transcriptional profiling using high-density microarrays provides unique data about disease mechanisms, drug responses, regulatory pathways, and gene function by comparing the level of mRNA transcribed in cells in a given pathologic state versus a control. This allows for the elucidation of complex pathophysiologic mechanisms directly at the gene expression level. ${ }^{8}$ We examined the cardiac and skeletal muscle gene expression profiles of patients undergoing cardiac surgery with CPB and cardioplegic arrest to improve the understanding of these modalities and provide a reference framework for the evaluation of prognostic gene markers and new intraoperative strategies.

\section{Methods}

\section{Tissue Samples}

After the protocol was approved by the research ethics committee of the Beth Israel Deaconess Medical Center and informed consent was obtained from the patients, samples of right atrium and skeletal muscle were collected immediately before and after CPB in 16 patients undergoing primary elective CABG. Samples were harvested with cold sharp dissection and handled in a nontraumatic fashion.

Right atrial samples consisted of nontraumatized tissue that was not involved in a purse string suture. Pre-CPB tissues were taken from the right atrial appendage before venous cannulation, and post-CPB atrial samples were harvested after the construction of a second purse string suture on the right atrial appendage and decannulation. Skeletal muscle samples were taken from the left intercostal muscle bed, lateral to the internal thoracic artery harvest site. Atrial and skeletal muscle samples $(\sim 10 \times 10 \times 2 \mathrm{~mm})$ were snap-frozen in liquid nitrogen and immediately stored at $-80^{\circ} \mathrm{C}$.

To minimize baseline phenotypic variation, 5 patients aged between 55 and 75 years, who were normocholesterolemic, nonsmoking, nondiabetic, of the same ethnicity and gender, and who had similar procedural characteristics, were selected from the 16 subjects whose tissues were harvested; 10 atrial (ie, 5 pre- and 5 post-CPB and cardioplegic arrest) and 10 skeletal muscle (ie, 5
TABLE 1. Patient characteristics

\begin{tabular}{lc}
\hline & $\begin{array}{c}\text { Atrial myocardial (N = 5) } \\
\text { and skeletal muscle } \\
\text { (N = 5) samples* }\end{array}$ \\
\hline Age (yr) $\dagger$ & $66(55,72)$ \\
Men/women, n & $5 / 0$ \\
Hypertension, $\mathrm{n}$ & 5 \\
Current tobacco use, $\mathrm{n}$ & 0 \\
Cholesterol $>200 \mathrm{mg} / \mathrm{dL}, \mathrm{n}$ & 0 \\
Diabetes, $\mathrm{n}$ & 0 \\
Redo or combined cardiac procedure, $\mathrm{n}$ & 0 \\
Number of grafts performed $\dagger$ & $3(3,4)$ \\
Duration of CPB (min) $\dagger$ & $74(67,99)$ \\
Duration of cardioplegic arrest (min) $\dagger$ & $60(44,83)$ \\
Minimum CPB temperature $\left({ }^{\circ} \mathrm{C}\right) \dagger$ & $34(33,35)$ \\
Intraoperative blood transfusion, $\mathrm{n}$ & 0 \\
Postoperative length of stay (d) $\dagger$ & $6(4,9)$ \\
\hline
\end{tabular}

CPB, Cardiopulmonary bypass.

* One tissue sample was collected from each site before and after CPB in each patient.

tData expressed as median (minimum, maximum).

pre- and 5 post-CPB) samples obtained from these patients were therefore used for the microarrays analyses and the validation studies (Table 1).

\section{RNA Isolation}

Total RNA was isolated with a Trizol-based method, following the manufacturer's protocol (Gibco BRL, Rockville, Md), on samples of approximately $200 \mathrm{mg}$. Purification was achieved by passing the RNA samples through an RNAeasy column (Qiagen, Valencia, Calif). Residual DNA contamination was eliminated by DNase I treatment (Invitrogen, San Diego, Calif). RNA concentrations were determined by spectrophotometry; each sample yielded a minimum of $5 \mu \mathrm{g}$ of total RNA at a minimum concentration of 0.5 $\mu \mathrm{g} / \mu \mathrm{L}$ with an $\mathrm{A}_{260} / \mathrm{A}_{280}$ ratio ranging between 1.8 and 2.1.

\section{Microarray Processing}

cDNA was prepared according to protocols provided with the Affymetrix U95 GeneChip system (Affymetrix, Santa Clara, Calif). ${ }^{9}$ Five micrograms of total RNA were used in the first-strand cDNA synthesis with T7-d(T) $)_{24}$ primer (GGCCAGTGAATTGTAATACGACTCACTATAGGGAGGCGG-[dT $]_{24}$ ) and Superscript II (Gibco-BRL, Gaithersburg, Md). The second-strand cDNA synthesis was performed at $16^{\circ} \mathrm{C}$ by adding Escherichia coli DNA ligase, DNA polymerase I, and RNase $\mathrm{H}$ to the reaction, followed by T4 DNA polymerase to blunt the ends of newly synthesized cDNA. The cDNA was purified through phenol/chloroform and ethanol precipitation. By using a BioArray High Yield RNA Transcript Labeling Kit (Enzo Diagnostics, Farmingdale, $\mathrm{NY}$ ), the purified cDNA was incubated for 5 hours at $37^{\circ} \mathrm{C}$ in an in vitro transcription reaction to produce cRNA-labeled with biotin.

\section{Microarray Hibridization}

cRNA was fragmented by incubating in a buffer containing 200 $\mathrm{mmol} / \mathrm{L}$ Tris-acetate, $500 \mathrm{mmol} / \mathrm{L} \mathrm{KOAc}$, and $150 \mathrm{mmol} / \mathrm{L}$ 
$\mathrm{MgOAc}$ for 35 minutes at $94^{\circ} \mathrm{C}$. Fifteen micrograms of fragmented cRNA were mixed with eukaryotic hybridization controls (containing control cRNA and oligonucleotide B2) and hybridized with a pre-equilibrated human U95Av2 Affymetrix chip for 16 hours at $45^{\circ} \mathrm{C}$. The chips were washed in a fluidic station with low-stringency buffer $(6 \times$ standard saline phosphate with EDTA, $0.01 \%$ Tween 20 , and $0.005 \%$ antifoam) for 10 cycles and in highstringency buffer (100 mmol/L N-morpholino-ethanesulfonic acid, $0.1 \mathrm{~mol} / \mathrm{L} \mathrm{NaCl}$, and $0.01 \%$ Tween 20 ) for 4 cycles and stained with streptavidin phycoerythrin. This process was followed by incubation with normal goat immunoglobulin $\mathrm{G}$ and biotinylated mouse anti-streptavidin antibody, and restaining with streptavidin phycoerythrin.

\section{Statistical Analysis of Microarray Data}

The chips were scanned in an HP G2500A ChipScanner (Affymetrix) to detect hybridization signals. Image output files were visually examined for major chip defects and hybridization artifacts, and raw data were processed with GeneChip Microarray Analysis Suite 5.0 software (Affymetrix). The image from each chip was scaled such that the absolute signal intensities were adjusted to target intensity and reported as a non-negative numerical quantity. Data were imported into SAS version 7 (SAS, Cary, NC) for further analysis. Statistical techniques were separately applied to each type of tissue and involved tests that were paired for each patient.

Median post- to pre-CPB and cardioplegic arrest gene expression ratios were computed for each gene by using the relative signal intensities of the probe cells. Two statistical methods were combined to assess differential gene expression between the preand post-CPB and cardioplegic arrest tissues. ${ }^{10}$ To ensure a minimal number of false positives, only the probe sets commonly yielded by the 2 methods were included in the list of genes differentially expressed in post- versus pre-CPB and cardioplegic arrest samples.

Method 1 identified probe sets with significant intensity differences between pre- and post-CPB and cardioplegic arrest tissues. For each tissue type, a Wilcoxon signed-rank test was applied to the absolute signal intensities of each gene in the pre- versus post-CPB and cardioplegic arrest data set. The use of a nonparametric, paired Wilcoxon test was selected over that of a standard $t$ test to avoid a distributional assumption; ${ }^{1,12}$ the threshold was set at a $P$ value of .0253 or less, which corresponds to the lowest possible $P$ value in the particular experimental setting of the study (observed when 5 of 5 patients show a similar pattern of differential expression). By using this $P$ value, the number of genes that could have reached significance by chance alone was determined and compared with the number of observed significant genes, and a signal-to-noise ratio was computed.

Method 2 determined the differentiability of a probe set by its signal intensity fold change. Nonparametric determination of genes with significantly different fold changes between the preand post-CPB and cardioplegic arrest states was performed by computing and ordering by increasing size the median relative signal intensities of each probe set. A particular gene was considered to be up-regulated after CPB and cardioplegic arrest in a given tissue type if a median fold change of $4(4: 1)$ or more was observed in post- versus pre-CPB and cardioplegic arrest samples.
Conversely, a gene was considered down-regulated if the median fold change of post- versus pre-CPB and cardioplegic arrest samples was $0.25(1: 4)$ or less. Only genes identified by both methods were considered to be differentially expressed and were reported.

\section{Microarray Validation}

Northern analysis. cDNA probes of human DUSP1 and CYR61 (2 significantly altered genes), and GADP and $\beta$-actin (2 genes that were not altered) were labeled with $\alpha^{32} \mathrm{P}$-dCTP (New England Nuclear, Boston, Mass) using a random-priming labeling kit (Boehringer, Indianapolis, Ind) and purified from unincorporated nucleotides using G-50 Quick Spin Columns (Boehringer). The blots were hybridized overnight at $48^{\circ} \mathrm{C}$ using an Ambion Northern Max kit (Ambion, Austin, Tex). After hybridization, the blots were washed twice in $2 \times$ standard saline citrate with $0.1 \%$ sodium dodecyl sulfate (SDS) for 15 minutes each at room temperature and then washed twice in $0.1 \times$ standard saline citrate with $0.1 \%$ SDS for 15 minutes each at $60^{\circ} \mathrm{C}$. Autoradiography was performed for 20 hours at $-80^{\circ} \mathrm{C}$. Blots were analyzed after digitization and quantification of x-ray films with the Image-Quant software (Molecular Dynamic, Sunnyvale, Calif), quantified as a ratio of the gene mRNA to loading band density, and presented as a median (minimum, maximum) ratio of post- to pre-CPB and cardioplegic arrest mRNA expression.

Real-time polymerase chain reaction. Reverse transcription was performed on $2 \mu \mathrm{g}$ of total RNA isolated from atrial and skeletal muscle tissues, using an oligo dT primer (Invitrogen) for 90 minutes at $37^{\circ} \mathrm{C}$. DUSP1 primers were forward-TGGAGGAAGGGTGTTTGTCC, reverse-CAAGGCAGATGGTGGCTGA, and TaqMan-FAM-CTGCCAGGCAGGCATTTCCCGTAMRA (Applied Biosystems, Foster City, Calif). CYR61 primers were forward-ATCAAGGACCCCATGGAGG, reverseAGGCATCGAATCCCAGCTC, and TaqMan-FAM-CCAGGACGGCCTCCTTGGCAA-TAMRA (Applied Biosystems). Amplification conditions were as follows: stage 1,1 cycle for 2 minutes at $50^{\circ} \mathrm{C}$; stage 2,1 cycle for 10 minutes at $95^{\circ} \mathrm{C}$; stage 3 , 40 cycles for 15 seconds at $95^{\circ} \mathrm{C}$ and 1 minute at $60^{\circ} \mathrm{C}$. Real-time detection was accomplished using SYBR Green I dye and fluorescence quantified with a GeneAmp Sequence Detection System (Applied Biosystems). All experiments were performed in duplicate, and post- to pre-CPB and cardioplegic arrest ratios of polymerase chain reaction (PCR) products are presented as median (minimum, maximum) ratios.

In situ hybridization. Probes for human DUSP1 and CYR61 were generated from PCR fragments containing T3 (forward) and $\mathrm{T} 7$ (reverse) promoter sequences incorporated in the amplicon. Frozen sections of $10 \mu \mathrm{m}$ were cut in a cryostat and captured onto microscope slides. Sections were fixed and acetylated, and hybridized at $68^{\circ} \mathrm{C}$ to the probe for 3 nights. Hybridized probes were visualized using alkaline phosphatase-conjugated anti-DIG Fab fragments and 5-bromo-4-chloro-3-indolyl-phosphate/nitro blue tetrazolium substrate (Kierkegard and Perry, Gaithersburg, Md). Sections were rinsed several times in $100 \mathrm{mmol} / \mathrm{L}$ Tris, 150 $\mathrm{mmol} / \mathrm{L} \mathrm{NaCl}$, and $20 \mathrm{mmol} / \mathrm{L}$ EDTA $\mathrm{pH} 9.5$, and coverslipped with glycerol gelatin. Control sections were incubated in an identical concentration of the sense probe transcript. In situ hybridization autoradiographs were evaluated qualitatively in random highpower fields. 
TABLE 2. Genes exhibiting a fourfold or greater increase in expression in post- versus pre-cardiopulmonary bypass and cardioplegic areas atrial samples

\begin{tabular}{|c|c|c|c|c|}
\hline Gene name & Symbol & $\begin{array}{c}\text { GenBank } \\
\text { accession } \\
\text { number }\end{array}$ & $\begin{array}{l}\text { Expression } \\
\text { ratio } \\
\text { (post:pre)* }\end{array}$ & Function \\
\hline Proto-oncogene C-FOS & FOS & K00650 & $20: 1$ & Pro-apoptotic transcription factor, acting with JUN \\
\hline $\begin{array}{l}\text { Nuclear receptor subfamily } 4 \text {, } \\
\text { group } A \text {, member } 1\end{array}$ & NR4A1 & L13740 & $19: 1$ & Steroid receptor; regulates apoptosis \\
\hline Proto-oncogene JUN-B & JUNB & M29039 & $17: 1$ & $\begin{array}{l}\text { Similar to JUN; pro-apoptotic transcription factor acting with } \\
\text { FOS }\end{array}$ \\
\hline $\begin{array}{l}\text { Nuclear receptor subfamily } 4 \text {, } \\
\text { group } A \text {, member } 2\end{array}$ & NR4A2 & X75918 & $15: 1$ & $\begin{array}{l}\text { Coactivator of transcription; stimulated by PGE2, IL1B, and } \\
\text { TNF }\end{array}$ \\
\hline Dual-specificity phosphatase 1 & DUSP1 & X68277 & $12: 1$ & Regulator of the cellular response to stress \\
\hline $\begin{array}{l}\text { Zinc finger transcriptional } \\
\text { regulator }\end{array}$ & ZFP36 & M92843 & $10: 1$ & $\begin{array}{l}\text { Component of a negative feedback loop that interferes with } \\
\text { TNF production }\end{array}$ \\
\hline Transcription factor 8 & TCF8 & D15050 & $10: 1$ & Negative regulator of T-lymphocyte IL2 gene expression \\
\hline $\begin{array}{l}\text { Nuclear receptor subfamily } 4 \text {, } \\
\text { group } A \text {, member } 3\end{array}$ & NR4A3 & D78579 & $7: 1$ & Transcription factor \\
\hline Oncogene FOS-B & FOSB & L49169 & $7: 1$ & $\begin{array}{l}\text { Transcription factor; its truncated form may act to limit the } \\
\text { transcriptional effects of FOS and JUN }\end{array}$ \\
\hline $\begin{array}{l}\text { Solute carrier family } 2 \text {, member } \\
\quad 3\end{array}$ & SLC2A3 & M20681 & $6: 1$ & $\begin{array}{l}\text { Facilitation of glucose transport in tissues with a high glucose } \\
\text { demand }\end{array}$ \\
\hline Cellular oncogene C-FOS & FOS & V01512 & $6: 1$ & Pro-apoptotic transcription factor, acting with JUN \\
\hline Dual-specificity phosphatase 5 & DUSP5 & U15932 & $6: 1$ & $\begin{array}{l}\text { Protein-tyrosine phosphatase induced by serum stimulation } \\
\text { and heat shock }\end{array}$ \\
\hline $\begin{array}{l}\text { MADS box transcription } \\
\text { enhancer factor } 2\end{array}$ & MEF2C & S57212 & $6: 1$ & Transcription factor \\
\hline Cyclin dependent kinase-like 1 & CDKL1 & X6358 & $6: 1$ & Cell cycle regulator \\
\hline Small inducible cytokine A2 & SCYA2 & M26683 & $6: 1$ & Chemotactic factor for monocytes; regulated by NF-kappa-B \\
\hline Oncogene MYC & MYC & V00568 & $5: 1$ & Transcription factor \\
\hline B-cell translocation gene 2 & BTG2 & U72649 & $5: 1$ & Cell cycle regulator in response to DNA damage \\
\hline T-cell cytokine receptor & TCCR & AF053004 & $5: 1$ & $\begin{array}{l}\text { Class I cytokine receptor; involved in the early immune } \\
\text { response }\end{array}$ \\
\hline $\begin{array}{l}\text { Plasminogen activator receptor, } \\
\text { urokinase-type }\end{array}$ & PLAUR & U09937 & $5: 1$ & Involved in neutrophil recruitment \\
\hline Activating transcription factor 3 & ATF3 & L19871 & $5: 1$ & Transcription factor \\
\hline Early growth response 2 & EGR2 & J04076 & $5: 1$ & Transcription factor \\
\hline Argininemia gene & ARG1 & M14502 & $5: 1$ & Hydrolyzes arginine to urea and omithine in the urea cycle \\
\hline Sortilin 1 & SORT1 & X98248 & $5: 1$ & Involved in sorting proteins to endosomal vesicles and golgi \\
\hline E-selectin & SELE & M24736 & $4: 1$ & Adhesion of leukocytes to cytokine-stimulated endothelial cells \\
\hline Kappa casein & CSN10 & M73628 & $4: 1$ & Unknown \\
\hline Cytokine GRO2 & GR02 & M36820 & $4: 1$ & $\begin{array}{l}\text { Chemotactic agent for polymorphonuclear leukocytes; } \\
\text { regulated by IL1B, TNF, and LPS }\end{array}$ \\
\hline $\begin{array}{l}\text { Cysteine-rich angiogenic inducer } \\
61\end{array}$ & CYR61 & Y11307 & $4: 1$ & $\begin{array}{l}\text { Immediate-early transcription factor; promotes adhesion of } \\
\text { endothelial cells, chemotaxis, and angiogenesis }\end{array}$ \\
\hline S100 calcium-binding protein A4 & S100A4 & M80563 & $4: 1$ & $\begin{array}{l}\text { Elevated serum levels have been correlated with cerebral } \\
\text { damage after cardiopulmonary bypass and circulatory arrest }\end{array}$ \\
\hline Histone H1F3 & H1F3 & M60747 & $4: 1$ & $\begin{array}{l}\text { Basic nuclear proteins responsible for the nucleosome } \\
\text { structure }\end{array}$ \\
\hline Fibronectin 1 & FN1 & AJ001487 & $4: 1$ & $\begin{array}{l}\text { Stimulates endocytosis and clearance of C1q-coated material } \\
\text { such as immune complexes, cellular debris, or particulate } \\
\text { material from the circulation }\end{array}$ \\
\hline mRNA export protein 1 & RAE1 & U85943 & $4: 1$ & Nucleocytoplasmic transport of mRNAs \\
\hline $\begin{array}{l}\text { Cyclin-dependent kinase } \\
\text { inhibitor } 1 \mathrm{~A}\end{array}$ & CDKN1A & U03106 & $4: 1$ & Associated with apoptosis of human endothelial cells \\
\hline
\end{tabular}

$n F$, Nuclear factor; $I L$, interleukin; $T N F$, tumor necrosis factor; $L P S$, Lipopolysaccharide.

${ }^{*}$ Median expression ratio of post-CPB versus pre-CPB samples. 
TABLE 3. Genes exhibiting a fourfold or greater decrease in expression in post- versus pre-cardiopulmonary bypass and cardioplegic arrest atrial samples

\begin{tabular}{|c|c|c|c|c|}
\hline Gene name & Symbol & $\begin{array}{c}\text { GenBank } \\
\text { accession } \\
\text { number }\end{array}$ & $\begin{array}{l}\text { Expression } \\
\text { ratio } \\
\text { (post:pre)* }\end{array}$ & Function \\
\hline Immunoglobulin gamma 3 & IGHG3 & Y14737 & $1: 47$ & Heavy chain immunoglobulin \\
\hline Annexin A8 & ANXA8 & X16662 & $1: 23$ & Vascular anticoagulant beta; function in heart unknown \\
\hline $\begin{array}{l}\text { Immunoglobulin kappa constant } \\
\text { region }\end{array}$ & IGKC & M63438 & $1: 18$ & Light chain immunoglobulin \\
\hline $\begin{array}{l}\text { Immunoglobulin lambda constant } \\
\text { region } 1\end{array}$ & IGLC1 & M18645 & $1: 16$ & Light chain immunoglobulin \\
\hline Haptoglobin & HP & X00442 & $1: 16$ & $\begin{array}{l}\text { Binds free hemoglobin and protects against bacterial } \\
\text { growth }\end{array}$ \\
\hline Immunoglobulin mu & IGHM & X67301 & $1: 16$ & Heavy chain immunoglobulin \\
\hline $\begin{array}{l}\text { Immunoglobulin lambda-like light } \\
\text { chain } 3 \text { (incomplete sequence) }\end{array}$ & - & A1932613 & $1: 9$ & Light chain immunoglobulin \\
\hline Keratin 7 & KRT7 & AJ238246 & $1: 7$ & Unknown \\
\hline $\begin{array}{l}\text { Protein tyrosine phosphatase, } \\
\text { non-receptor, type } 2\end{array}$ & PTPN2 & M25393 & $1: 7$ & Regulator of cell growth and differentiation \\
\hline Centrin 1 & CETN1 & U03270 & $1: 6$ & $\begin{array}{l}\text { Involved in centrosome segregation and microtubule } \\
\text { severing }\end{array}$ \\
\hline $\begin{array}{l}\text { Immunoglobulin alpha constant } \\
\text { heavy chain } 2\end{array}$ & IGHA2 & S71043 & $1: 6$ & Heavy chain immunoglobulin \\
\hline Properdin factor B & $\mathrm{BF}$ & L15702 & $1: 6$ & Serine protease; C3 proaccelerator \\
\hline Hexosaminidase B & HEXB & M23294 & $1: 6$ & Ganglioside breakdown \\
\hline Cyclin E2 & CCNE2 & AF091433 & $1: 5$ & DNA synthesis initiator \\
\hline $\begin{array}{l}\text { Tumor necrosis factor receptor } \\
\text { superfamily, member } 7\end{array}$ & TNFRSF7 & M63928 & $1: 5$ & Cell growth, differentiation, and apoptosis \\
\hline $\begin{array}{l}\text { Immunoglobulin lambda constant } \\
\text { region }\end{array}$ & IGLC & $X 57809$ & $1: 5$ & Light chain immunoglobulin \\
\hline Kallikrein 11 & KLK11 & AB012917 & $1: 5$ & Serine protease \\
\hline RAS inhibitor & RIN1 & L36463 & $1: 4$ & $\begin{array}{l}\text { Inhibitor of RAS-mediated cell growth and } \\
\text { differentiation }\end{array}$ \\
\hline $\begin{array}{l}\text { Carcinoembryonic antigen- } \\
\quad \text { related cell adhesion molecule }\end{array}$ & CEACAM1 & X16354 & $1: 4$ & Member of the immunoglobulin superfamily \\
\hline Haptoglobin-related protein & HPR & X89214 & $1: 4$ & Similar to haptoglobulin \\
\hline Immunoglobulin J chain & IGJ & Al660656 & $1: 4$ & $\begin{array}{l}\text { Synthesized in B lymphocytes; links immunoglobulin } \\
\text { monomers }\end{array}$ \\
\hline $\begin{array}{l}\text { RAL guanine nucleotide } \\
\text { dissociation stimulator }\end{array}$ & RALGDS & AB002349 & $1: 4$ & RAS-related GTPases; cell signaling \\
\hline $\begin{array}{l}\text { Phosphoenolpyruvate } \\
\text { carboxykinase } 2 \\
\text { (mitochondrial) }\end{array}$ & PCK2 & X92720 & $1: 4$ & Gluconeogenesis \\
\hline Mast cell carboxypeptidase A & MC-CPA & M73720 & $1: 4$ & Unknown \\
\hline DMX-like 1 protein & DMXL1 & AJ005821 & $1: 4$ & Protein-protein interactions and regulation \\
\hline $\begin{array}{l}\text { Neuronal nicotinic acetylcholine } \\
\text { receptor alpha-7 subunit }\end{array}$ & CHRNA7 & X70297 & $1: 4$ & $\begin{array}{l}\text { Alpha } 7 \text { subunit of the neuronal nicotinic acetylcholine } \\
\text { receptor }\end{array}$ \\
\hline
\end{tabular}

*Median expression ratio of post-CPB versus pre-CPB samples.

Immunoblotting. Total lysate from atrial and skeletal muscle tissues was obtained by 30 -second homogenization on ice in lysis buffer containing $1 \%$ NP-40, $0.5 \%$ sodium deoxycholate, $0.1 \%$ SDS, and a protease inhibitor, and centrifuged at 12,000g for 10 minutes at $4{ }^{\circ} \mathrm{C}$ to separate soluble from insoluble proteins. The supernatant protein concentration was measured spectrophotometrically at $595 \mathrm{~nm}$ wavelength using a BCA protein assay kit (Pierce, Rockford, Ill). Total protein was fractionated on $10 \%$ SDS-polyacrylamide gel electrophoresis and transferred to a poly- vinylidene difluoride membrane (Millipore, Bedford, Mass) using a semi-dry transfer apparatus. Membranes were stained with Ponceau $\mathrm{S}$ and incubated with $5 \%$ nonfat dry milk in $50 \mathrm{mmol} / \mathrm{L}$ Tris- $\mathrm{HCl}, \mathrm{pH} 8.0,100 \mathrm{mmol} / \mathrm{L} \mathrm{NaCl}$, and $0.1 \%$ Tween 20 (TBST) buffer for 1 hour at room temperature. Membranes were incubated with antibodies (in 2.5\% nonfat dry milk in TBST) (anti-DUSP1 $1: 1000[\mathrm{v} / \mathrm{v}]$ dilution, rabbit polyclonal $1: 1000[\mathrm{v} / \mathrm{v}]$, or antiCYR61 1/1000 [v/v] dilution), and rabbit polyclonal (Santa Cruz Biotechnology, Santa Cruz, Calif) for 2 hours. After washing with 
TABLE 4. Genes exhibiting a fourfold or greater increase in expression in post- versus pre-cardiopulmonary bypass skeletal muscle samples

\begin{tabular}{|c|c|c|c|c|}
\hline Gene name & Symbol & $\begin{array}{c}\text { GenBank } \\
\text { accession } \\
\text { number }\end{array}$ & $\begin{array}{l}\text { Expression } \\
\text { ratio } \\
\text { (post:pre)* }\end{array}$ & Function \\
\hline Interleukin 6 & IL6 & X04430 & $20: 1$ & $\begin{array}{l}\text { Mediates leukocytosis, thrombosis, and lymphocyte } \\
\text { activation }\end{array}$ \\
\hline Interleukin 8 & IL8 & M28130 & $18: 1$ & Mediates neutrophils chemotaxis and migration \\
\hline $\begin{array}{l}\text { Tumor necrosis factor receptor } \\
\text { superfamily, member 11B }\end{array}$ & TNFRSF11B & AB008822 & $13: 1$ & Secreted by T cells; mediates inflammation \\
\hline $\begin{array}{l}\text { Nuclear receptor subfamily } 4 \text {, group } \\
\text { A, member } 3\end{array}$ & NR4A3 & D78579 & $9: 1$ & Transcription factor \\
\hline $\begin{array}{l}\text { Nuclear receptor subfamily } 4 \text {, group } \\
\text { A, member } 1\end{array}$ & NR4A1 & L13740 & $8: 1$ & Steroid receptor; regulates apoptosis \\
\hline Transcription factor 8 & TCF8 & D15050 & $6: 1$ & Negative regulator of T-lymphocyte IL2 gene expression \\
\hline Early growth response 3 & EGR3 & X63741 & $6: 1$ & $\begin{array}{l}\text { Immediate-early transcription activator; induced by } \\
\text { stress or injury }\end{array}$ \\
\hline Cholesterol 25-hydroxylase & $\mathrm{CH} 25 \mathrm{H}$ & AF059214 & $6: 1$ & Regulation of cholesterol and lipid metabolism \\
\hline T-cell receptor interacting molecule & TRIM & AJ224878 & $6: 1$ & $\begin{array}{l}\text { Associates with the TCR-CD3 complex in T Iymphocytes } \\
\text { after T-cell activation }\end{array}$ \\
\hline Endothelin 1 & EDN1 & J05008 & $6: 1$ & Potent vasoconstrictor produced by endothelial cells \\
\hline $\begin{array}{l}\text { Transforming growth factor-beta } \\
\text { receptor, type } 1\end{array}$ & TGFBR1 & AF054598 & $6: 1$ & Proliferation and differentiation of fibroblasts \\
\hline Interleukin 13 & IL13 & U31120 & $6: 1$ & $\begin{array}{l}\text { Expressed in activated human T lymphocytes; synergizes } \\
\text { with IL2 in regulating inflammatory and immune } \\
\text { responses }\end{array}$ \\
\hline Ephrin B2 & EFNB2 & U81262 & $6: 1$ & Angiogenesis and vascular remodeling \\
\hline Activating transcription factor 2 & ATF2 & L05515 & $5: 1$ & Transcription factor \\
\hline T-cell tyrosine kinase EMT & EMT & L10717 & $5: 1$ & TCR-mediated CD4 stimulation \\
\hline Oncogene JUN-B & JUNB & X51345 & $5: 1$ & Pro-apoptotic transcription factor acting with FOS \\
\hline TAF13 RNA polymerase II & TAF13 & X84003 & $5: 1$ & $\begin{array}{l}\text { Mediates the function of gene promoters and } \\
\text { transcriptional activators }\end{array}$ \\
\hline ATPase, class 1 , type $8 \mathrm{~A}$, member 2 & ATP8A2 & Al017981 & $5: 1$ & Transport of ions across cell membranes \\
\hline Solute carrier family 2 , member 3 & SLC2A3 & M20681 & $5: 1$ & $\begin{array}{l}\text { Facilitated glucose transporter in tissues that exhibit a } \\
\text { high glucose demand }\end{array}$ \\
\hline $\begin{array}{l}\text { Platelet glycoprotein } 1 \mathrm{~B} \text {, alpha } \\
\text { polypeptide }\end{array}$ & GP1BA & J02940 & $5: 1$ & Platelet adhesion \\
\hline $\begin{array}{l}\text { Signal-induced proliferation- } \\
\text { associated gene } 1\end{array}$ & SIPA1 & AB005666 & $5: 1$ & $\begin{array}{l}\text { Involved with IL2 in mitogen-induced cell cycle } \\
\text { progression }\end{array}$ \\
\hline CD44 surface antigen & CD44 & L05424 & $5: 1$ & $\begin{array}{l}\text { Matrix adhesion and migration of lymphocytes from the } \\
\text { bloodstream to lymphatic tissues }\end{array}$ \\
\hline $\begin{array}{l}\text { Inhibitor of kappa light chain gene } \\
\text { enhancer in B cells, epsilon }\end{array}$ & IKBE & U91616 & $5: 1$ & Inhibits NF-kappa-B transcription \\
\hline TEC protein tyrosine kinase & TEC & D29767 & $4: 1$ & Expressed in myeloid, B-, and T-cell lineages \\
\hline Dual-specificity phosphatase 2 & DUSP2 & L11329 & $4: 1$ & A MAP kinase phosphatase \\
\hline Runt-related transcription factor 1 & RUNX1 & X90976 & $4: 1$ & Transcription factor \\
\hline TAF1 RNA polymerase II & TAF1 & X07024 & $4: 1$ & $\begin{array}{l}\text { Mediates the function of gene promoters and } \\
\text { transcriptional activators }\end{array}$ \\
\hline Protease inhibitor 9 & PI9 & U71364 & $4: 1$ & Serine protease inhibitor \\
\hline $\begin{array}{l}\text { Protein-tyrosine phosphatase, } \\
\text { receptor-type, J }\end{array}$ & PTPRJ & U10886 & $4: 1$ & Modulation of biologic cascades \\
\hline $\begin{array}{l}\text { Activated leukocyte cell adhesion } \\
\text { molecule }\end{array}$ & ALCAM & Y10183 & $4: 1$ & T-cell receptor; involved in cell adhesion interactions \\
\hline RAS-associated protein RAB27A & RAB27A & U57094 & $4: 1$ & Platelet vesicular transport \\
\hline Interleukin 1-beta & IL1B & M15330 & $4: 1$ & $\begin{array}{l}\text { Cytokine involved in inflammation, cell-cell signaling, } \\
\text { signal transduction, and the antimicrobial response }\end{array}$ \\
\hline
\end{tabular}

*Median expression ratio of post-CPB versus pre-CPB samples. 
TABLE 5. Genes exhibiting a fourfold or greater decrease in expression in post- versus pre-cardiopulmonary bypass skeletal-muscle samples

\begin{tabular}{|c|c|c|c|c|}
\hline Gene name & Symbol & $\begin{array}{c}\text { GenBank } \\
\text { accession } \\
\text { number }\end{array}$ & $\begin{array}{l}\text { Expression } \\
\text { ratio (post: } \\
\text { pre) }\end{array}$ & Function \\
\hline Factor H-related gene 2 & FHR2 & X64877 & $1: 7$ & $\begin{array}{l}\text { Structurally and immunologically related to complement } \\
\text { factor } \mathrm{H}\end{array}$ \\
\hline T-box 1 & TBX1 & AF012130 & $1: 5$ & Transcription factor \\
\hline $\begin{array}{l}\text { Protein phosphatase 1, regulatory } \\
\text { subunit } 3 A\end{array}$ & PPP1R3A & AF024578 & $1: 5$ & $\begin{array}{l}\text { Centrally involved in carbohydrate and lipid metabolism; } \\
\text { down-regulation or mutation can cause severe } \\
\text { insulin resistance }\end{array}$ \\
\hline RAS-related protein $2 \mathrm{~A}$ & RAP2A & X12534 & $1: 5$ & Unknown \\
\hline Growth/differentiation factor 8 & GDF8 & AF104922 & $1: 4$ & Maintenance of skeletal muscle homeostasis \\
\hline Chorionic gonadotropin, beta chain & CGB & J00117 & $1: 4$ & Function in skeletal muscle unclear \\
\hline $\begin{array}{l}\text { Cleavage and polyadenylation } \\
\text { specificity factor } 6\end{array}$ & CPSF6 & X67337 & $1: 4$ & Processing of primary mRNA transcripts \\
\hline
\end{tabular}

*Median expression ratio of post-CPB versus pre-CPB samples.

TBST, the membranes were incubated for 1 hour in $2.5 \%$ nonfat dry milk in TBST diluted with anti-rabbit immunoglobulin G (Jackson Immunolabs, West Grove, Pa), both at 1:3000 (v/v) dilution conjugated to horseradish peroxidase. Peroxidase activity was visualized using enhanced chemiluminescence and exposed to $\mathrm{X}$-ray films (Amersham, Arlington Heights, Ill). Post-CPB to pre$\mathrm{CPB}$ and cardioplegic arrest ratios of protein density are presented as median (minimum, maximum) ratios.

\section{Reporting and Functional Classification}

Genes identified to be significant by microarray analysis with both statistical methods were the object of a literature search and reported, along with their GenBank accession number, according to the current nomenclature of Online Mendelian Inheritance in Man. ${ }^{13}$ Functional inferences outlined in the right column of Tables 2 to 5 are based on the authors' interpretation of literature referenced in Online Mendelian Inheritance in Man pertaining to each gene.

\section{Results}

\section{Patient Characteristics}

Table 1 displays the clinical characteristics of the patients whose tissues were used for the microarray and validation studies. All subjects were Caucasian men aged between 55 and 72 years old, without significant comorbidity, who electively underwent 3 or 4 CABGs with mildly hypothermic CPB and antegrade intermittent (every 20 minutes) hyperkalemic cold blood cardioplegia. Antifibrinolytic drugs were not administered.

\section{Microarray Signal-to-Noise Characteristics}

Of 12,625 genes examined, 1,106 and 974 genes were identified by method 1 to have a $P$ value of .0253 or less for their differential expression in post- versus pre-CPB and cardioplegic arrest cardiac and skeletal muscle tissues, respectively (Figure 1). The projected number of genes that could have reached significance because of chance alone was 320 for each tissue type, corresponding to a signal-tonoise ratio of 3.46 in cardiac and 3.04 in skeletal muscle microarrays.

\section{Identification of Significantly Altered Genes}

Genes whose expression was found to be significantly altered by using both methods are reported in Tables 2 to 5 . Of these, there were 32 up-regulated and 26 down-regulated genes in the heart (Tables 2 and 3), and 32 and 7 such genes in the skeletal muscle (Tables 4 and 5). Most up-regulated atrial genes were transcription factors, and most downregulated atrial genes were immunoglobulins. In skeletal muscle, transcription factors also represented a large proportion of the up-regulated genes, but no specific functional pattern was observed in the down-regulated genes.

\section{Validation}

DUSP1. The microarray results for DUSP1 revealed increased expression (median 12-fold; 3.2, 27.1; $P=.025$ ) in the atrium, but no significant change in the skeletal muscle after CPB and cardioplegic arrest (median 1.6-fold; $0.3,6.5 ; P=.68)$. Validation studies revealed the median mRNA expression of DUSP1 in post- versus pre-CPB atrial samples to be increased 3-fold $(1.7,7.1 ; P=.025)$ and 6.4-fold $(1.5,16.8 ; P=.05)$ by Northern blotting and real-time PCR, respectively, with a change in protein level of 4.6-fold $(1.3,5.7 ; P=.05)$ by immunoblotting (Figure 2). In skeletal muscle, DUSP1 was not significantly upregulated, with a median differential mRNA expression of 1.6-fold $(1.2,4.6 ; P=.08)$ and 8.2-fold $(2.5,23.3 ; P=.08)$ by Northern blotting and PCR, respectively, and a protein level change of 2.1 -fold $(1.1,3.0 ; P=.68)$ by immunoblotting. In situ hybridization revealed a striking increase in DUSP1 expression in atrial tissues, particularly localized to 

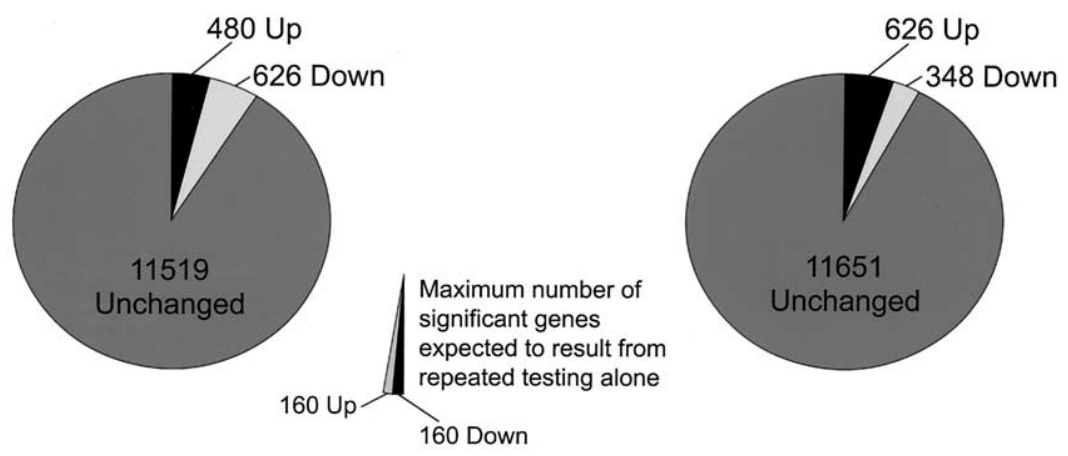

Figure 1. Genes that were differentially expressed with a $\boldsymbol{P}$ value of .0253 or less after cardiopulmonary bypass (CPB) and antegrade cold blood cardioplegia in cardiac (left) and skeletal muscle tissues (right) (of 12,625 genes examined). These genes and the number expected to result from repeat testing alone were identified by method 1 described in the text.

\section{DUSP1 CYR61}
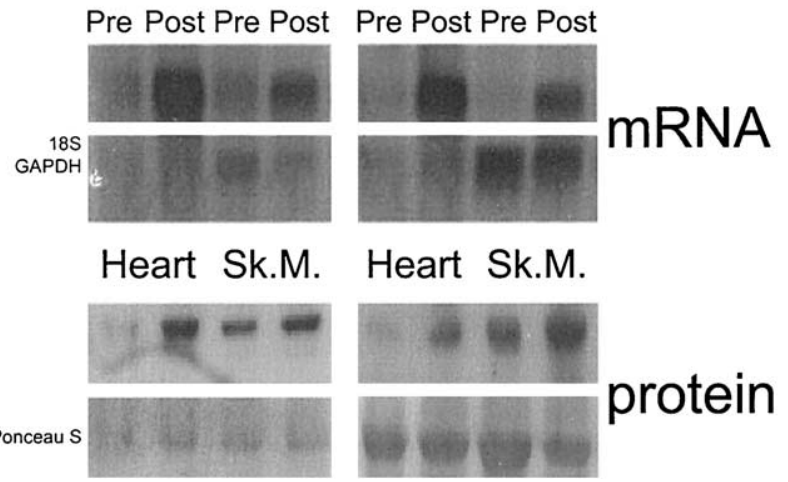

Figure 2. Northern and Western blot analyses of mRNA expression and protein level of DUSP1 and CYR61 in atrial (heart) and skeletal muscle (Sk.M.) tissues, pre- and post-CPB and cardioplegia. mRNA expression and protein levels of DUSP1 and CYR61 were significantly increased in post- versus pre-CPB atrial samples, but not in the skeletal muscle.

the endothelium and myocytes (Figure 3). No increase in expression was detected in skeletal muscle samples.

CYR61. The microarray results for CYR61 revealed increased expression in the atrium (median 4-fold; 1.5, 12.1; $P=.025)$, but no significant change in the skeletal muscle after CPB and cardioplegic arrest (median 4-fold; 0.8, 8; $P$ $=.68$ ). In concordance with these results, the median expression of CYR61 in post- versus pre-CPB atrial samples was 2.5 -fold $(1.8,5.3 ; P=.025)$ and 6.2 -fold $(1.4,15.4 ; P$ $=.025$ ) by Northern blotting and real-time PCR, respectively (Figure 2). The amount of protein was also increased 2.7 -fold $(1.5,3.2 ; P=.05)$ in atrial tissues. In the skeletal muscle, CYR61 was not significantly up-regulated, with a median differential expression of 1 -fold $(1,2.3 ; P=.16)$ by Northern blotting, 4.9-fold $(1,15.9 ; P=.08)$ by PCR, and 2.7-fold by immunoblotting $(0.9,3.2 ; P=.68)$.
GAPD and $\beta$-Actin. Microarray results revealed the differential expression of these genes not to be significantly altered after CPB and cardioplegic arrest in both atrial and skeletal muscle tissues. On Northern blotting, the median differential expression of post-CPB and cardioplegic arrest to pre-CPB samples was 1.2 and 1.1 for GAPD, and 1.0 and 1.1 for $\beta$-actin in atrial and skeletal muscle samples, respectively $(P=$ not significant $)$.

\section{Discussion}

In this study, the gene expression profile of atrial and skeletal muscle from selected, phenotypically similar patients undergoing cardiac surgery with CPB and cardioplegic arrest was determined. The microarray-based method was feasible and validated with conventional quantification techniques. A large number of genes showing altered transcription with a subthreshold $P$ value were identified, well in excess of the number projected to have reached this $P$ value threshold by multiple testing alone. We determined that $\mathrm{CPB}$ and cardioplegic arrest do not cause an indiscriminate change in gene expression, but rather induce a distinct pattern of changes in specific pathways such as up-regulation of inflammation/transcription activators, apoptotic genes, and stress genes, as well as down-regulation of immunoglobulin genes. These results allow a better understanding of the pathophysiology of CPB and cardioplegic arrest on cardiac and peripheral tissues and introduce a new method for evaluating modalities aimed at minimizing the adverse effects of $\mathrm{CPB}$, cardioplegia, and cardiac surgery by directly measuring the overall gene expression profile and response of target groups of genes.

Several of the genes found to be differentially expressed in this study have been implicated in the pathophysiology of $\mathrm{CPB}$ and cardioplegia in humans. FOS and JUN were found to be up-regulated in subendocardial myocytes and endothelial cells of right atrial samples after cold crystalloid cardioplegia. ${ }^{14,15}$ Nuclear factor-kappa B activation has 


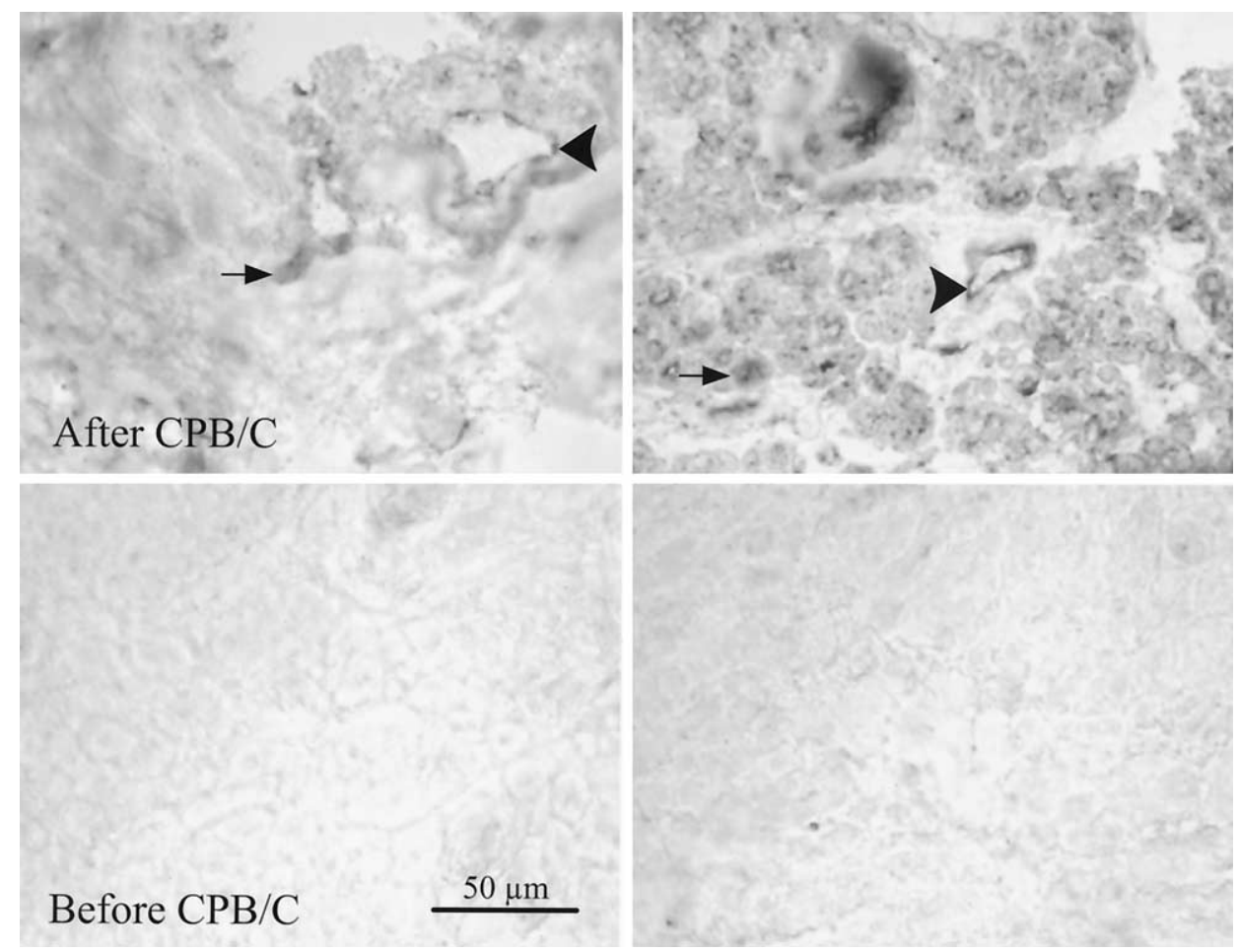

Figure 3. Representative in situ hybridization images of DUSP1, pre- and post-CPB in atrial tissues. A post-CPB and cardioplegic arrest increase in DUSP1 expression was localized to the endothelium (arrowheads) and myocytes (small arrows).

been proposed as an inducer of transcription in right atrial tissue exposed to ischemia-reperfusion, ${ }^{16}$ and endothelin 1 receptor blockade has been associated with minimization of left ventricular dysfunction after CPB and cardioplegic arrest in swine. ${ }^{17}$ A number of neutrophil adhesion molecules such as CD11 and CD44 have been differentially expressed, ${ }^{18,19}$ and serum levels of IL6, IL8, TNF, IL1B, SCYA2, SELE, and IL13, and CD44 levels have been shown to be increased after $\mathrm{CPB} .{ }^{20-26}$ Elevated serum levels of S100 have been correlated with cerebral damage after CPB and circulatory arrest. ${ }^{27}$

This study also identifies multiple genes that had not been implicated in the pathophysiology of CPB and cardioplegic arrest, such as DUSP1, TCF8, CYR61, SLC2A3, MYC, TCCR, BTG2, TRIM, ALCAM, EFNB2, and many others (Tables 2-5). Although the clinical significance of these new findings is still unknown, further research may characterize the expression of these genes, quantify polymorphisms, and examine their correlation with surgical outcome.

\section{Limitations}

Microarray techniques, despite their immense potential to improve disease understanding, have inherent shortcomings related to the selection of tissues, lack of standardized methods for the statistical analysis of data, presentation of vast amounts of results, and generalizability of the findings. ${ }^{28}$ Microarray technology also does not allow for the determination of absolute expression patterns and provides a measure of transcribed mRNA rather than a functional gene product. We have attempted to limit these shortcomings by selecting phenotypically and procedurally similar subjects, combining nonparametric statistical methods to minimize false positives, validating the expression patterns, protein levels, and origin of select genes with conventional molecular techniques, and suggesting a potential functional role for genes whose expression was most strikingly altered.

For ethical reasons, we used human atrial biopsies rather than ventricular biopsies to examine the cardiac gene expression changes that result from CPB and cardioplegic arrest. Atrial sampling involves little or no intrinsic risk of morbidity, provides full-thickness samples, and is clinically reproducible. CPB and antegrade cold blood cardioplegia distribute to the right atrium. ${ }^{29}$ Although atrial myocardium differs from ventricular myocardium with respect to the relative percentage of myocytic, endothelial, connective, and neural elements, these cell types are present in each tissue type and may demonstrate ultrastructural changes in disease. ${ }^{30,31}$ 


\section{Conclusion}

We believe that this study allows a better understanding of the cardiac and systemic effects of CPB and cardioplegic arrest. We anticipate that these data will serve as a starting point for functional studies and provide a framework for the use of gene expression approaches to better understand and minimize the morbid effects of cardiac surgery on cardiac and peripheral tissues.

\section{References}

1. Sellke FW, Tofukuji M, Stamler A, et al. Beta-adrenergic regulation of the cerebral microcirculation after hypothermic cardiopulmonary bypass. Circulation. 1997;96:II304-10.

2. Stamler A, Wang SY, Aguirre DE, et al. Cardiopulmonary bypass alters vasomotor regulation of the skeletal muscle microcirculation. Ann Thorac Surg. 1997;64:460-5.

3. Stamler A, Wang SY, Aguirre DE, et al. Effects of pentastarchdeferoxamine conjugate on lung injury after cardiopulmonary bypass. Circulation. 1996;94:II358-63.

4. Friedman M, Wang SY, Stahl GL, et al. Altered beta-adrenergic and cholinergic pulmonary vascular responses after total cardiopulmonary bypass. J Appl Physiol. 1995;79:1998-2006.

5. Wang SY, Friedman M, Franklin A, et al. Myogenic reactivity of coronary resistance arteries after cardiopulmonary bypass and hyperkalemic cardioplegia. Circulation. 1995;92:1590-6.

6. Sellke FW, Shafique T, Ely DL, et al. Coronary endothelial injury after cardiopulmonary bypass and ischemic cardioplegia is mediated by oxygen-derived free radicals. Circulation. 1993;88:II395-400.

7. Metais C, Li J, Simons M, et al. Serotonin-induced coronary contraction increases after blood cardioplegia-reperfusion: role of COX-2 expression. Circulation. 1999;100:II328-34.

8. Duggan DJ, Bittner M, Chen Y, et al. Expression profiling using cDNA microarrays. Nat Genet. 1999;21:10-4.

9. Iacobuzio-Donahue CA, Maitra A, Shen-Ong GL, et al. Discovery of novel tumor markers of pancreatic cancer using global gene expression technology. Am J Pathol. 2002;160:1239-49.

10. Haslett JN, Sanoudou D, Kho AT, et al. Gene expression comparison of biopsies from Duchenne muscular dystrophy (DMD) and normal skeletal muscle. Proc Natl Acad Sci U S A. 2002;99:15000-5.

11. Wilcoxon F. Individual comparisons by ranking methods. Biometrics. 1946;1:80-83.

12. Liu WM, Mei R, Di X, et al. Analysis of high density expression microarrays with signed-rank call algorithms. Bioinformatics. 2002; 18:1593-9.

13. Available at http://www.ncbi.nlm.nih.gov/entrez/query.fcgi?CMD= Limits\&DB $=$ omim.

14. Aebert H, Cornelius T, Birnbaum DE, et al. Induction of early immediate genes and programmed cell death following cardioplegic arrest in human hearts. Eur J Cardiothorac Surg. 1997;12:261-7.

15. Nelson DP, Wechsler SB, Miura T, et al. Myocardial immediate early gene activation after cardiopulmonary bypass with cardiac ischemiareperfusion. Ann Thorac Surg. 2002;73:156-62.

16. Canty TG Jr, Boyle EM Jr, Farr A, et al. Oxidative stress induces NF-kappaB nuclear translocation without degradation of IkappaBalpha. Circulation. 1999;100:II361-4.

17. Pearl JM, Nelson DP, Wagner CJ, et al. Endothelin receptor blockade reduces ventricular dysfunction and injury after reoxygenation. Ann Thorac Surg. 2001;72:565-70.

18. Ilton MK, Langton PE, Taylor ML, et al. Differential expression of neutrophil adhesion molecules during coronary artery surgery with cardiopulmonary bypass. J Thorac Cardiovasc Surg. 1999;118:930-7.

19. Toft $\mathrm{P}$, Nielsen $\mathrm{CH}$, Tonnesen $\mathrm{E}$, et al. Changes in adhesion molecule expression and oxidative burst activity of granulocytes and monocytes during open-heart surgery with cardiopulmonary bypass compared with abdominal surgery. Eur J Anaesthesiol. 1998;15:345-53.

20. Brull DJ, Montgomery HE, Sanders J, et al. Interleukin-6 gene $-174 \mathrm{~g}>\mathrm{c}$ and $-572 \mathrm{~g}>\mathrm{c}$ promoter polymorphisms are strong predictors of plasma interleukin-6 levels after coronary artery bypass surgery. Arterioscler Thromb Vasc Biol. 2001;21:1458-63.

21. Chello M, Mastroroberto P, Quirino A, et al. Inhibition of neutrophil apoptosis after coronary bypass operation with cardiopulmonary bypass. Ann Thorac Surg. 2002;73:123-9.

22. Gormley SM, McBride WT, Armstrong MA, et al. Plasma and urinary cytokine homeostasis and renal dysfunction during cardiac surgery. Anesthesiology. 2000;93:1210-6.

23. Kawahito K, Adachi H, Ino T. Influence of surgical procedures on interleukin-6 and monocyte chemotactic and activating factor responses: CABG vs. valvular surgery. J Interferon Cytokine Res. 2000;20: $1-6$.

24. Burns SA, Newburger JW, Xiao M, et al. Induction of interleukin-8 messenger RNA in heart and skeletal muscle during pediatric cardiopulmonary bypass. Circulation. 1995;92:II315-21.

25. Matata BM, Galinanes M. Cardiopulmonary bypass exacerbates oxidative stress but does not increase proinflammatory cytokine release in patients with diabetes compared with patients without diabetes: regulatory effects of exogenous nitric oxide. J Thorac Cardiovasc Surg. 2000;120:1-11.

26. Nathan N, Preux PM, Feiss P, et al. Plasma interleukin-4, interleukin10 , and interleukin-13 concentrations and complications after coronary artery bypass graft surgery. J Cardiothorac Vasc Anesth. 2000;14:15660.

27. LeMaire SA, Bhama JK, Schmittling ZC, et al. S100beta correlates with neurologic complications after aortic operation using circulatory arrest. Ann Thorac Surg. 2001;71:1913-8.

28. King HC, Sinha AA. Gene expression profile analysis by DNA microarrays: promise and pitfalls. JAMA. 2001;286:2280-8.

29. Stirling MC, McClanahan TB, Schott RJ, et al. Distribution of cardioplegic solution infused antegradely and retrogradely in normal canine hearts. J Thorac Cardiovasc Surg. 1989;98:1066-76.

30. Gorza L, Mercadier JJ, Schwartz K, et al. Myosin types in the human heart. An immunofluorescence study of normal and hypertrophied atrial and ventricular myocardium. Circ Res. 1984;54:694-702.

31. Hoffmann U, Axmann C, Grisk A. Myosin isoenzymes in normal and hypertrophied human hearts. Biomed Biochim Acta. 1986;45:985-996. 